\title{
Liouville theorems for fully nonlinear elliptic equations on spherically symmetric Riemannian manifolds
}

\author{
Fabio Punzo
}

\begin{abstract}
We prove Hadamard and Liouville type theorems for viscosity supersolutions to fully nonlinear elliptic equations on spherically symmetric complete noncompact Riemannian manifolds.

Mathematics Subject Classification (2010). 35B50, 35B53, 35D40, 35J60, 58J05.

Keywords. Liouville theorem, Hadamard theorem, Sub-supersolutions, Ricci curvature, Maximum pinciples, Radial solutions.
\end{abstract}

\section{Introduction}

We investigate Liouville property for continuous viscosity supersolutions to fully nonlinear uniformly elliptic equations of the types:

$$
\begin{array}{ll}
\mathcal{P}_{\lambda, \Lambda}^{-}\left(D^{2} u\right)=0 & \text { in } M, \\
\mathcal{P}_{\lambda, \Lambda}^{+}\left(D^{2} u\right)=0 & \text { in } M
\end{array}
$$

and

$$
F\left(x, D^{2} u\right)+h(x) u^{p}=0 \text { in } M .
$$

Here $\mathcal{P}_{\lambda, \Lambda}^{-}, \mathcal{P}_{\lambda, \Lambda}^{+}$are the extremal Pucci operators $(0<\lambda \leq \Lambda), F$ is a uniformly elliptic operator, $h$ is a nonnegative continuous function defined in $M$, $p>0$; furthermore, $M$ is a complete noncompact spherically symmetric manifold (or model manifold), equipped with the metric

$$
d s^{2}=d \rho^{2}+\sigma^{2}(\rho) \gamma_{i j}(\theta) d \theta^{i} d \theta^{j},
$$

where $(\rho, \theta)$ are polar geodesic coordinates with respect to a pole $o \in M$, $\gamma_{i j}(\theta) d \theta^{i} d \theta^{j}$ is the standard metric on $\mathbb{S}^{N-1}$ and $\sigma \in C^{\infty}([0, \infty) ;[0, \infty))$ satisfies

$$
\sigma(0)=0, \quad \sigma^{\prime}(0)=1, \quad \sigma>0 \quad \text { in }(0, \infty) .
$$


Liouville type theorems have been largely investigated for supersolutions to equation

$$
\Delta u=0 \text { in } \mathcal{M}
$$

where $\mathcal{M}$ is a complete noncompact Riemannian manifold (see, e.g., [8,9] and references therein); moreover, divergence form nonlinear elliptic equations has also been considered (see [15]). Let us mention that Liouville theorems have been also established on the Heisenberg group for semilinear elliptic equations (see $[2])$.

On the other hand, Liouville type results have been proved for viscosity supersolutions to equation (1.1), (1.2), or (1.3) in the special case $M=\mathbb{R}^{N}$, (that is $\sigma(\rho)=\rho(\rho \geq 0)$ in $(1.4))$ (see $[4,5])$.

Define the geodesic ball with center in $o$ and radius $r>0$

$$
B_{r}:=\{x \in M \mid \rho(x)<r\} .
$$

The area of the geodesic sphere $\partial B_{r}=\{x \in M \mid \rho(x)=r\}$ is

$$
S(r)=\omega_{N} \sigma^{N-1}(r),
$$

where $\omega_{N}$ is the area of the unit sphere of $\mathbb{R}^{N}$.

As is well-known any bounded supersolution to equation

$$
\Delta u=0 \text { in } M
$$

is constant if and only if

$$
\int_{1}^{\infty} \frac{d \xi}{S(\xi)}=\frac{1}{\omega_{N}} \int_{1}^{\infty} \frac{d \xi}{[\sigma(\xi)]^{N-1}}=\infty
$$

(see [8]). When such Liouville property holds, $M$ is said parabolic; otherwise, it is said non-parabolic.

Let

$$
\begin{aligned}
& \alpha \equiv \alpha_{\lambda, \Lambda, N}:=\frac{\lambda}{\Lambda}(N-1), \\
& \beta \equiv \beta_{\lambda, \Lambda, N}:=\frac{\Lambda}{\lambda}(N-1) .
\end{aligned}
$$

When $M=\mathbb{R}^{N}$, in [5] it is proved that any viscosity supersolution bounded from below to equation (1.2) is constant, if

$$
\alpha \leq 1
$$

For further purposes, observe that in this case

$$
\int_{1}^{\infty} \frac{d \xi}{[S(\xi)]^{\frac{\lambda}{\Lambda}}}=\frac{1}{\omega_{N}^{\lambda / \Lambda}} \int_{1}^{\infty} \frac{d \xi}{[\sigma(\xi)]^{\alpha}}=\frac{1}{\omega_{N}^{\lambda / \Lambda}} \int_{1}^{\infty} \frac{d \xi}{\xi^{\alpha}}=\infty .
$$

Moreover, under proper hypotheses on $h$ and $p$, it is shown that any nonnegative viscosity supersolution to equation (1.3) vanishes identically, if

$$
\beta>1 \text {. }
$$


Observe that in this case

$$
\int_{1}^{\infty} \frac{d \xi}{[S(\xi)]^{\frac{\Lambda}{\lambda}}}=\frac{1}{\omega_{N}^{\Lambda / \lambda}} \int_{1}^{\infty} \frac{d \xi}{[\sigma(\xi)]^{\beta}}=\frac{1}{\omega_{N}^{\Lambda / \lambda}} \int_{1}^{\infty} \frac{d \xi}{\xi^{\beta}}<\infty .
$$

The aim of this paper is to generalize previous Liouville type results for viscosity supersolutions to equations (1.1), (1.2) or (1.3), when $M$ is a model manifold. More precisely, we shall prove that:

(i) (see Theorem 4.1) any viscosity supersolution bounded from below to equation (1.2) is constant, if

$$
\sigma^{\prime} \geq 0 \text { in }\left(\rho_{0}, \infty\right) \text { for some } \rho_{0} \geq 0
$$

and

$$
\int_{1}^{\infty} \frac{d \xi}{[S(\xi)]^{\frac{\lambda}{\Lambda}}}=\infty
$$

(ii) (see Theorem 5.1) any nonnegative viscosity supersolution to equation (1.3) is identically zero, if Ricci curvature of $M$ is nonnegative and

$$
\int_{1}^{\infty} \frac{d \xi}{[S(\xi)]^{\frac{\Lambda}{\lambda}}}<\infty
$$

under proper hypotheses on $h$ and $p$.

Observe that, when $M=\mathbb{R}^{N}$, our results are in agreement with those given in [5] (see $(1.10),\left(H_{1}\right),(1.11)$ and $\left.\left(H_{2}\right)\right)$.

Moreover, note that if $\lambda=\Lambda=1$ equations (1.1) and (1.2) recast into equation (1.7); in this case by our methods we can recover the Liouville property given in [8] for equation (1.7) (see Remark 4.3).

In the sequel a crucial role will be played by classical radial solutions to the following equations:

$$
\mathcal{P}_{\lambda, \Lambda}^{-}\left(D^{2} u\right)=0 \quad \text { in } M \backslash \bar{B}_{\rho}
$$

and

$$
\mathcal{P}_{\lambda, \Lambda}^{+}\left(D^{2} u\right)=0 \quad \text { in } M \backslash \bar{B}_{\rho_{0}},
$$

with $\rho_{0} \geq 0$ given in $(C)$. Clearly, for $\rho_{0}=0$ equations (1.12), (1.13) become:

$$
\mathcal{P}_{\lambda, \Lambda}^{-}\left(D^{2} u\right)=0 \quad \text { in } M \backslash\{o\}
$$

and

$$
\mathcal{P}_{\lambda, \Lambda}^{+}\left(D^{2} u\right)=0 \quad \text { in } M \backslash\{o\} .
$$

In view of the metric (1.4), the construction of these radial solutions is more technical than the Euclidean case (see Sects. 2.2, 2.3). However, once we have obtained such classical radial solutions, we can follow the same line of arguments of [5] to prove the Liouville type theorems. In fact, at first we deduce Hadamard three cycles results for the functions

$$
m(\rho):=\min _{\bar{B}_{\rho}} u \quad(\rho>0),
$$


and

$$
M(\rho):=\max _{\bar{B}_{\rho}} u \quad(\rho>0),
$$

that also have an independent interest (see Theorems 3.1 and 3.2). These results are proved by using classical radial solutions to equations (1.12) and (1.13), and then applying comparison principles. Then the Liouville theorem for equations (1.2) and (1.1) is deduced by these Hadamard results, applying maximum principles.

Furthermore, in the proof of the Liouville property for equation (1.3) we use the fact that the function

$$
\rho \mapsto \frac{m(\rho)}{\int_{\rho}^{\infty} \frac{d \xi}{[\sigma(\xi)]^{\beta}}} \quad(\rho>0)
$$

is increasing in $(0, \infty)$ (see Corollary 3.4); this will be deduced from Hadamard type results. Since such a function depends on $\sigma$, the proof is more technical than the Euclidean case; however, in view of the hypothesis that the Ricci curvature is nonnegative, using the so-called Laplacian comparison principle (see (5.51)), we can get the conclusion following the same strategy as in [5]; indeed, this assumption on the Ricci curvature is essential in order to the result holds true (see Remark 5.2).

One can expect that by similar methods also fully nonlinear elliptic equations with gradient term with linear growth can be treated (see [4] for $M=$ $\mathbb{R}^{N}$ ); however, we shall only consider equations (1.1), (1.3) for the sake of simplicity.

The paper is organized as follows. In Sect. 2 we recall some preliminaries from Riemannian Geometry and some auxiliary results concerning fully nonlinear equations, Pucci operators and radial solutions to equations (1.1) and (1.2). In Sect. 3 we show Hadamard type results, which will used to prove Liouville Theorems stated in Sect. 4 for equations (1.2) and (1.1), and in Sect. 5 for equation (1.3).

\section{Mathematical framework}

\subsection{Preliminaries from Riemannian Geometry}

In this Subsection we recall some notions from Riemannian Geometry that will be used in the sequel.

Let $M$ be a smooth $N$-dimensional manifold equipped with the metric tensor $g$; denote by $\left(g_{i j}\right)_{i, j}$ the components of the tensor $g$ in local coordinates $x^{1}, \ldots, x^{N}$, and by $\left(g^{i j}\right)_{i, j}$ the inverse matrix of $\left(g_{i j}\right)_{i, j}$. Let $x \in M$; let $T_{x} M$ be the tangent space at $x, T M$ be the tangent bundle of $M, T_{x}^{*} M$ be the cotangent space at $x, T^{*} M$ be the cotangent bundle of $M, \Gamma(T M)$ denote the vector space of smooth sections of $T M$.

Recall that the Laplace-Beltrami operator on $(M, g)$ is given by:

$$
\Delta=\frac{1}{\sqrt{\operatorname{det} g}} \frac{\partial}{\partial x^{i}}\left(\sqrt{\operatorname{det} g} g^{i j} \frac{\partial}{\partial x^{j}}\right) ;
$$


moreover, for any $f \in C^{1}(M ; \mathbb{R})$ the gradient of $f$ is:

$$
(\nabla f)^{i}=g^{i j} \frac{\partial f}{\partial x^{j}}
$$

The Levi-Civita connection $D$ of the metric $g$ is given by

$$
D_{\frac{\partial}{\partial x^{i}}} \frac{\partial}{\partial x^{j}}=\Gamma_{i j}^{k} \frac{\partial}{\partial x^{k}},
$$

where

$$
\Gamma_{i j}^{k}:=\frac{1}{2} g^{k l}\left(\frac{\partial g_{j l}}{\partial x^{i}}+\frac{\partial g_{i l}}{\partial x^{j}}-\frac{\partial g_{i j}}{\partial x^{l}}\right)
$$

are the Christoffel symbols.

We also recall that the Hessian of $f \in C^{2}(M ; \mathbb{R})$ is the symmetric endomorphism of $T M$ defined by

$$
D^{2} u(X):=D_{X} \nabla u \quad(X \in \Gamma(T M)),
$$

or its associated symmetric bilinear form on $T M$ defined by

$$
\left(D^{2} f\right)(X, Y):=X(Y(f))-D_{X} Y(f) \quad(X, Y \in \Gamma(T M)) .
$$

We have:

$$
\left(D^{2} f\right)(X, Y)=\left\langle D_{X}(\nabla f), Y\right\rangle=D_{X}\left(D_{Y}(f)\right) \quad(X, Y \in \Gamma(T M)) .
$$

Also, in local coordinates there holds:

$$
\left(D^{2} f\right)_{i j}=\left(D^{2} f\right)\left(\frac{\partial}{\partial x^{i}}, \frac{\partial}{\partial x^{j}}\right)=\frac{\partial^{2} f}{\partial x^{i} \partial x^{j}}-\Gamma_{i j}^{k} \frac{\partial f}{\partial x^{k}} .
$$

We have:

$$
\Delta f=\operatorname{tr}\left[\left(D^{2} f\right)\left(\frac{\partial}{\partial x^{i}}, \frac{\partial}{\partial x^{j}}\right)\right]=\sum_{i, j=1}^{N} g^{i j}\left(D^{2} f\right)\left(\frac{\partial}{\partial x^{i}}, \frac{\partial}{\partial x^{j}}\right) ;
$$

here and hereafter tr denotes the trace operator.

Suppose that $(M, g)$ has a point $o$ with an empty cut locus; then $o$ is called the pole of $M$ and $M$ a manifold with a pole. Therefore, to every point $x \in M \backslash\{o\}$ there correspond a polar radius $\rho:=\operatorname{dist}(x, o)$ and a polar angle $\theta \in \mathbb{S}^{N-1}$ such that the shortest geodesics from $o$ to $x$ starts at $o$ with direction $\theta$. Thus the polar coordinates $(\rho, \theta)$ are defined in $M \backslash\{o\}$. The Riemannian metric $g$ in $M \backslash\{0\}$, in the polar coordinates, has the form

$$
d s^{2}=d \rho^{2}+A_{i j}(\rho, \theta) d \theta^{i} d \theta^{j},
$$

where $\theta^{1}, \ldots \theta^{N-1}$ are coordinates on $\mathbb{S}^{N-1}$ and $\left(A_{i j}\right)_{i, j=1, \ldots, N}$ is a positive definite matrix.

A manifold with a pole is called a model manifold or a spherically symmetric manifold, if

$$
A_{i j}(\rho, \theta) d \theta^{i} d \theta^{j}=\sigma^{2}(\rho) \gamma_{i j}(\theta) d \theta^{i} d \theta^{j}
$$

(see (1.4)). By hypothesis (1.5), the metric

$$
d s^{2}=d \rho^{2}+\sigma^{2}(\rho) \gamma_{i j} d \theta^{i} d \theta^{j}
$$

can be smoothly extended from $M \backslash\{o\}$ to the whole of $M$. 
Since we shall consider complete noncompact Riemannian model manifolds, we assume that $\sigma \in C^{\infty}([0, \infty))$; more in general, it could be considered $\sigma \in C^{\infty}\left(\left[0, R_{0}\right)\right)$, for some $R_{0}>0$.

As special cases, observe that if $\sigma(\rho)=\rho(\rho \in[0, \infty))$, then $M=\mathbb{R}^{N}$; whereas, if $\sigma(\rho)=\sinh \rho(\rho \in[0, \infty))$, then $M=\mathbb{H}^{N}$.

In the sequel we shall always take the following system of local coordinates:

$$
x^{1} \equiv \rho,\left(x^{2}, \ldots, x^{N}\right) \equiv\left(\theta^{1}, \ldots, \theta^{N-1}\right),
$$

SO

$$
g_{11}=1, \quad g_{1 j}=g_{j 1}=0 \quad \text { if } j=2, \ldots, N,
$$

and

$$
g_{i j}=\sigma^{2}(\rho) \gamma_{i-1 j-1}(\theta) \quad \text { if } i, j=2, \ldots, N .
$$

Hence we have:

$$
g^{11}=1, \quad g^{1 j}=g^{j 1}=0 \quad \text { if } j=2, \ldots, N,
$$

and

$$
g^{i j}=\frac{1}{[\sigma(\rho)]^{2}} \gamma^{i-1 j-1}(\theta) \quad \text { if } i, j=2, \ldots, N ;
$$

here $\left(\gamma^{i j}\right)_{i, j}$ is the inverse matrix of $\left(\gamma_{i j}\right)_{i, j}$.

The volume $V(r)$ of the geodesic ball $B(o, r)$ is

$$
V(r):=\int_{0}^{r} S(\xi) d \xi=\omega_{N} \int_{0}^{r} \sigma^{N-1}(\xi) d \xi
$$

The Laplace-Beltrami operator on $M$ can be written as

$$
\Delta=\frac{\partial^{2}}{\partial \rho^{2}}+(N-1) \frac{\sigma^{\prime}}{\sigma} \frac{\partial}{\partial \rho}+\frac{1}{[\sigma(\rho)]^{2}} \Delta_{\theta}=\frac{\partial^{2}}{\partial \rho^{2}}+\frac{S^{\prime}}{S} \frac{\partial}{\partial \rho}+\frac{1}{[\sigma(\rho)]^{2}} \Delta_{\theta},
$$

where $\Delta_{\theta}$ is the Laplace-Beltrami operator on $\mathbb{S}^{N-1}$.

Denote by $\omega$ the plane of $T_{x} M$ with basis $\left(\frac{\partial}{\partial \rho}, X\right)$, where $X$ is a unit vector orthogonal to $\frac{\partial}{\partial \rho}$. Furthermore, denote by $K_{\omega}(x)$ the sectional curvature of the plane $\omega$, and by $\operatorname{Ric}_{o}(x)$ the Ricci curvature of $M$ at $x$ in the radial direction $\frac{\partial}{\partial \rho}$. For every $x \equiv(\rho, \theta) \in M$ we have:

$$
\begin{aligned}
K_{\omega}(x) & =-\frac{\sigma^{\prime \prime}(\rho)}{\sigma(\rho)} \\
\operatorname{Ric}_{o}(x) & =-(N-1) \frac{\sigma^{\prime \prime}(\rho)}{\sigma(\rho)} .
\end{aligned}
$$

Recall that (see $[8,9]$ ) every Riemannian manifold has a unique heat kernel $K \in C^{\infty}(M \times M \times(0, \infty))$, namely

$$
\left(e^{t \Delta} \phi\right)(x)=\int_{M} K(x, y, t) \phi(y) d \nu(y) \quad(x \in M)
$$


for every $\phi \in L^{2}(M)$, where $e^{t \Delta}$ is the semigroup generated by the operator $\Delta$ on $L^{2}(M)$ and $\nu$ is the Riemannian measure. Properties of the heat kernel $K$ are well-known (see $[8,9])$.

The Green function is defined as follows:

$$
G(x, y):=\int_{0}^{\infty} K(x, y, t) d t \quad((x, y) \in M \times M)
$$

and it takes values in $(0, \infty]$. It is direct to see that $G\left(x_{0}, y_{0}\right)<\infty$ for some $x_{0}, y_{0} \in M, x_{0} \neq y_{0}$ if and only if $G(x, y)<\infty$ for any $x, y \in M, x \neq y$.

If

$$
G(x, y)<\infty \quad \text { for any } \quad x, y \in M, x \neq y
$$

then

$$
\Delta G(x, y)=0 \text { for any } x \in M \backslash\{y\} .
$$

Note that when $M$ is a model manifold, then we have:

$$
G(x, o)=\int_{\rho(x)}^{\infty} \frac{d \xi}{S(\xi)} \quad(x \in M \backslash\{o\}),
$$

here $\rho(x)=\operatorname{dist}(x, o)$.

\subsection{Nonlinear operators on Riemannian manifolds}

Let $\mathcal{E}$ be the set of symmetric endomorphisms defined in $T M$. Let $\mathcal{E}$ be the set of symmetric endomorphisms defined in $T M$. We assume that $F: M \times \mathcal{E} \rightarrow \mathbb{R}$ is a continuous function with

$$
F(x, 0)=0 \text { for all } x \in M .
$$

Moreover, we suppose that $F$ is uniformly elliptic with ellipticity constants $0<\lambda \leq \Lambda$, that is

$$
\lambda \operatorname{tr}(P) \leq F(x, A+P)-F(x, A) \leq \Lambda \operatorname{tr}(P)
$$

for all $x \in M, A, P \in \mathcal{E}$ with $P \geq 0$ (i.e., $P$ is positive semidefinite).

In local coordinates $x^{1}, \ldots, x^{N}$, as usual, we can express any $A \in \mathcal{E}$ by a symmetric $N \times N$-matrix, putting $a_{i j}:=g\left(A\left(\frac{\partial}{\partial x^{i}}\right), \frac{\partial}{\partial x^{j}}\right)$. Hence $F$ can be regarded as a real valued continuous function defined in $M \times \mathcal{S}^{N}$, where $\mathcal{S}^{N}$ denotes the set of symmetric $N \times N$ matrices.

Set

$$
\mathcal{A}_{\lambda, \Lambda}:=\left\{\left.A \in \mathcal{E}|\lambda| X\right|^{2} \leq g(A(X), X) \leq \Lambda|X|^{2} \text { for any } X \in \Gamma(T M)\right\},
$$

where $|X|^{2}:=g(X, X) \quad(X \in \Gamma(T M))$. For any $B \in \mathcal{E}$ define

$$
\mathcal{P}_{\lambda, \Lambda}^{-}(B):=\inf _{A \in \mathcal{A}_{\lambda, \Lambda}} \operatorname{tr}(A \circ B), \mathcal{P}_{\lambda, \Lambda}^{+}(B):=\sup _{A \in \mathcal{A}_{\lambda, \Lambda}} \operatorname{tr}(A \circ B) .
$$

It is easily seen that

$$
\begin{aligned}
& \mathcal{P}_{\lambda, \Lambda}^{-}(B):=\lambda \sum_{e_{i}>0} e_{i}+\Lambda \sum_{e_{i}<0} e_{i}, \\
& \mathcal{P}_{\lambda, \Lambda}^{+}(B):=\Lambda \sum_{e_{i}>0} e_{i}+\lambda \sum_{e_{i}<0} e_{i},
\end{aligned}
$$


where $e_{i}(i=1, \ldots, N)$ denote the eigenvalues of $B \in \mathcal{E}$, with respect to the metric $g$. Furthermore,

$$
\mathcal{P}_{\lambda, \Lambda}^{+}(B)=-\mathcal{P}_{\lambda, \Lambda}^{-}(-B) \quad(B \in \mathcal{E}) .
$$

Clearly, when $\lambda=\Lambda=1$, then

$$
\mathcal{P}_{\lambda, \Lambda}^{+}(B)=\mathcal{P}_{\lambda, \Lambda}^{-}(B)=\operatorname{tr}(B) \text {. }
$$

Let us mention that equations involving Pucci operators on Riemannian manifolds have been considered also in [17], where Harnack estimates are investigated; moreover, linear nondivergence form elliptic equations on Riemannian manifolds have been addressed in $[3,10,16]$, where several qualitative properties of solutions are shown.

In local coordinates $x^{1}, \ldots, x^{N}$, if we express any $A \in \mathcal{E}$ by a matrix of $\mathcal{S}^{N}$, we get:

$$
\mathcal{P}_{\lambda, \Lambda}^{-}(B):=\inf _{A \in \tilde{\mathcal{A}}_{\lambda, \Lambda}} \operatorname{tr}(A B), \mathcal{P}_{\lambda, \Lambda}^{+}(B):=\sup _{A \in \tilde{\mathcal{A}}_{\lambda, \Lambda}} \operatorname{tr}(A B)
$$

where

$$
\tilde{\mathcal{A}}_{\lambda, \Lambda}:=\left\{A \in \mathcal{S}^{N} \mid \lambda\|X\|^{2} \leq\langle A X, X\rangle \leq \Lambda\|X\|^{2} \quad \text { for any } X \in \mathbb{R}^{N}\right\},
$$

$\|\cdot\|$ and $\langle\cdot, \cdot\rangle$ denoting the Euclidean norm and the Euclidean scalar product, respectively.

Moreover, it is direct to see that

$$
\begin{aligned}
& \mathcal{P}_{\lambda, \Lambda}^{-}(B)=\lambda \operatorname{tr}\left(B^{+}\right)-\Lambda \operatorname{tr}\left(B^{-}\right), \\
& \mathcal{P}_{\lambda, \Lambda}^{+}(B)=\Lambda \operatorname{tr}\left(B^{+}\right)-\lambda \operatorname{tr}\left(B^{-}\right)
\end{aligned}
$$

if $B^{+}, B^{-} \in \mathcal{S}^{N}, B^{+} \geq 0, B^{-} \geq 0$ with $B=B^{+}-B^{-}, B^{+} B^{-}=O$, where

$$
\operatorname{tr} A=g^{i j} a_{i j} \quad\left(A \equiv\left(a_{i j}\right) \in \mathcal{S}^{N}\right) .
$$

Furthermore, if $F$ satisfies conditions (2.27) and (2.28), then

$$
\mathcal{P}_{\lambda, \Lambda}^{-}(A) \leq F(x, A) \leq \mathcal{P}_{\lambda, \Lambda}^{+}(A)
$$

for all $x \in M$ and $A \in \mathcal{S}^{N}$.

Let us give the following

Definition 2.1. A function $u \in C(M ; \mathbb{R})$ is a viscosity supersolution (subsolution) of equation

$$
F\left(x, D^{2} u\right)=0 \text { in } M
$$

if, for all $\zeta \in C^{2}(M ; \mathbb{R})$ and $x_{0} \in M$ such that $u-\zeta$ has a local minimum (maximum) at $x_{0}$, there holds

$$
F\left(x_{0}, D^{2} \zeta\left(x_{0}\right)\right) \leq(\geq) 0 .
$$

We say that $u$ is a viscosity solution, if $u$ is both a supersolution and a subsolution.

In the sequel we shall use the comparison principle and the strong maximum principles contained in next propositions (see $[1,13]$ ). 
Proposition 2.2. Let $\Omega \subset M$ be a bounded open set and let $f \in C(\Omega)$. If $u_{1}$ and $u_{2}$ are respectively a sub- and a supersolution either to equation

$$
\mathcal{P}_{\lambda, \Lambda}^{-}\left(D^{2} u\right)=f \quad \text { in } \Omega
$$

or to

$$
\mathcal{P}_{\lambda, \Lambda}^{+}\left(D^{2} u\right)=f \quad \text { in } \Omega
$$

and if

$$
u_{1} \leq u_{2} \quad \text { on } \partial \Omega
$$

then

$$
u_{1} \leq u_{2} \quad \text { in } \Omega .
$$

Proposition 2.3. Let $\Omega \subset M$ be a bounded open set and let $u$ be a subsolution (supersolution) either to equation

$$
\mathcal{P}_{\lambda, \Lambda}^{-}\left(D^{2} u\right)=0 \quad \text { in } \Omega
$$

or to

$$
\mathcal{P}_{\lambda, \Lambda}^{+}\left(D^{2} u\right)=0 \quad \text { in } \Omega .
$$

If $u$ attains its minimum (maximum) at an interior point of $\Omega$, then $u$ is constant.

\subsection{Classical radial solutions}

Now we look for classical radial solutions to equations (1.12) and (1.13). To do this we need some preliminary lemmas. Let us start with next

Lemma 2.4. Let $\phi \in C^{2}\left(M \backslash \bar{B}_{\rho_{0}} ; \mathbb{R}\right)$ be a radial function, namely $\phi=\phi(\rho)$. Then

$$
\begin{aligned}
& \left(D^{2} \phi\right)_{11}=\phi^{\prime \prime}(\rho), \quad\left(D^{2} \phi\right)_{1 i}=\left(D^{2} \phi\right)_{i 1}=0 \quad \text { for any } i=2, \ldots, N \\
& \left(D^{2} \phi\right)_{i j}=\phi^{\prime}(\rho) \sigma^{\prime}(\rho) \sigma(\rho) \gamma_{i-1 j-1}(\theta) \quad \text { for any } i, j=2, \ldots, N
\end{aligned}
$$

Proof. Since $\frac{\partial \phi(\rho)}{\partial \theta^{i}}=0\left(\rho>\rho_{0}\right)$ for any $i=1, \ldots, N-1$, and

$$
\Gamma_{11}^{1}=0, \Gamma_{i j}^{k}=\sigma^{\prime}(\rho) \sigma(\rho) \gamma_{i-1 j-1}(\theta) \quad \text { for any } k=1, \ldots, N ; i, j=2, \ldots, N,
$$

from (2.17) the thesis easily follows.

From Lemma 2.4 and (2.31)-(1.12), we get the following

Lemma 2.5. Let hypothesis $(C)$ be satisfied. Let $\phi \in C^{2}\left(M \backslash \bar{B}_{\rho_{0}} ; \mathbb{R}\right)$ be a radial function.

(i) If $\phi$ is concave and increasing in $\left(\rho_{0}, \infty\right)$, then

$$
\mathcal{P}_{\lambda, \Lambda}^{-}\left(D^{2} \phi(\rho)\right)=\Lambda \phi^{\prime \prime}(\rho)+\lambda(N-1) \frac{\sigma^{\prime}(\rho)}{\sigma(\rho)} \phi^{\prime}(\rho) \quad\left(\rho>\rho_{0}\right)
$$

and

$$
\left.\mathcal{P}_{\lambda, \Lambda}^{+}\left(D^{2} \phi(\rho)\right)\right)=\lambda \phi^{\prime \prime}(\rho)+\Lambda(N-1) \frac{\sigma^{\prime}(\rho)}{\sigma(\rho)} \phi^{\prime}(\rho) \quad\left(\rho>\rho_{0}\right) .
$$


(ii) If $\phi$ is convex and decreasing in $\left(\rho_{0}, \infty\right)$, then

$$
\mathcal{P}_{\lambda, \Lambda}^{-}\left(D^{2} \phi(\rho)\right)=\lambda \phi^{\prime \prime}(\rho)+\Lambda(N-1) \frac{\sigma^{\prime}(\rho)}{\sigma(\rho)} \phi^{\prime}(\rho) \quad\left(\rho>\rho_{0}\right)
$$

and

$$
\mathcal{P}_{\lambda, \Lambda}^{+}\left(D^{2} \phi(\rho)\right)=\Lambda \phi^{\prime \prime}(\rho)+\lambda(N-1) \frac{\sigma^{\prime}(\rho)}{\sigma(\rho)} \phi^{\prime}(\rho) \quad\left(\rho>\rho_{0}\right) .
$$

Proof. Put

$$
\begin{aligned}
& a_{1 j}=a_{j 1}=0 \text { for any } j=1, \ldots, N, \\
& a_{i j}:=\phi^{\prime}(\rho) \sigma^{\prime}(\rho) \sigma(\rho) \gamma_{i-1 j-1}(\theta) \text { for any } i, j=2, \ldots, N,
\end{aligned}
$$

and

$$
\begin{aligned}
& b_{11}:=-\phi^{\prime \prime}(\rho), b_{1 j}=b_{j 1}=0 \text { for any } j=2, \ldots, N ; \\
& b_{i j}:=0 \quad \text { for any } i, j=2, \ldots, N .
\end{aligned}
$$

Since $\sigma^{\prime} \geq 0, \phi^{\prime \prime} \leq 0, \phi^{\prime} \geq 0$ in $\left(\rho_{0}, \infty\right)$ and $\left(\gamma_{i j}\right)_{i, j}$ is positive definite, we can apply $(2.31)$ with $B^{+}:=\left(a_{i j}\right)_{i, j}, B^{-}:=\left(b_{i j}\right)_{i, j}$. Thus, by (2.33),

$$
\begin{aligned}
\mathcal{P}_{\lambda, \Lambda}^{-}\left(D^{2} \phi(\rho)\right) & =\lambda \operatorname{tr}\left(B^{+}\right)-\Lambda \operatorname{tr}\left(B^{-}\right)=\lambda g^{i j} a_{i j}-\Lambda g^{i j} b_{i j} \\
& =\lambda \frac{\gamma^{i-1 j-1}}{[\sigma(\rho)]^{2}} \phi^{\prime}(\rho) \sigma^{\prime}(\rho) \sigma(\rho) \gamma_{i-1 j-1}-\Lambda g^{11} b_{11} \\
& =\Lambda \phi^{\prime \prime}(\rho)+\lambda(N-1) \frac{\sigma^{\prime}(\rho)}{\sigma(\rho)} \phi^{\prime}(\rho) \quad\left(\rho>\rho_{0}\right),
\end{aligned}
$$

whence (2.35). Similarly, we can get (2.36)-(2.38).

Remark 2.6. If $\lambda=\Lambda=1$, from (2.23) and (2.30) we deduce that hypothesis $(C)$ in Lemma 2.5 can be removed. The same holds in all subsequent results, since condition $(C)$ is only used to apply Lemma 2.5 .

In the following two lemmas we exhibit classical radial solutions to equations (1.12) and (1.13). We shall consider the following condition:

$$
\int_{1}^{\infty} \frac{d \xi}{[S(\xi)]^{\frac{\lambda}{\Lambda}}}<\infty .
$$

Lemma 2.7. Let hypothesis $(C)$ be satisfied.

(i) Let $C_{1}>0, C_{2} \in \mathbb{R}$; then

$$
\phi_{1}(\rho):=C_{1} \int_{1}^{\rho} \frac{d \xi}{[\sigma(\xi)]^{\alpha}}+C_{2} \quad\left(\rho>\rho_{0}\right)
$$

is a solution to equation (1.12).

Moreover,

$$
\psi_{1}(\rho):=-\phi_{1}(\rho) \quad\left(\rho>\rho_{0}\right)
$$

is a solution to equation (1.13). 
(ii) Let assumption $\left(H_{3}\right)$ be satisfied. Let $C_{1}>0, C_{2} \in \mathbb{R}$; then

$$
\phi_{2}(\rho):=-C_{1} \int_{\rho}^{\infty} \frac{d \xi}{[\sigma(\xi)]^{\alpha}}+C_{2} \quad\left(\rho>\rho_{0}\right)
$$

is a solution to equation (1.12). Moreover,

$$
\psi_{2}(\rho):=-\phi_{2}(\rho) \quad\left(\rho>\rho_{0}\right)
$$

is a solution to equation (1.13).

Proof. Note that both $\phi_{1}$ and $\phi_{2}$ are concave and increasing in $\left(\rho_{0}, \infty\right)$; moreover, they solve

$$
\Lambda u^{\prime \prime}+\lambda(N-1) \frac{\sigma^{\prime}}{\sigma} u^{\prime}=0 \quad \text { in }\left(\rho_{0}, \infty\right) .
$$

By Lemma 2.5, $\phi_{1}$ and $\phi_{2}$ are solutions to equation (1.12). By (2.29) and Lemma 2.5, $\psi_{1}$ and $\psi_{2}$ are solutions to Eq. (1.13).

Lemma 2.8. Let hypothesis $(C)$ be satisfied.

(i) Let $C_{1}>0, C_{2} \in \mathbb{R}$; then

$$
\phi_{3}(\rho):=-C_{1} \int_{1}^{\rho} \frac{d \xi}{[\sigma(\xi)]^{\beta}}+C_{2} \quad\left(\rho>\rho_{0}\right)
$$

is a solution to equation (1.12). Moreover,

$$
\psi_{3}(\rho):=-\phi_{3}(\rho) \quad\left(\rho>\rho_{0}\right)
$$

is a solution to equation (1.13).

(ii) Let condition $\left(H_{2}\right)$ be satisfied. Let $C_{1}>0, C_{2} \in \mathbb{R}$; then

$$
\phi_{4}(\rho):=C_{1} \int_{\rho}^{\infty} \frac{d \xi}{[\sigma(\xi)]^{\beta}}+C_{2} \quad\left(\rho>\rho_{0}\right)
$$

is a solution to equation (1.12). Moreover,

$$
\psi_{4}(\rho):=-\phi_{4}(\rho) \quad\left(\rho>\rho_{0}\right)
$$

is a solution to equation (1.13).

Proof. Note that $\phi_{3}$ and $\phi_{4}$ are convex and decreasing in $\left(\rho_{0}, \infty\right)$; furthermore, they solve

$$
\lambda u^{\prime \prime}+\Lambda(N-1) \frac{\sigma^{\prime}}{\sigma} u^{\prime}=0 \quad \text { in }\left(\rho_{0}, \infty\right) .
$$

By Lemma 2.5, $\phi_{3}$ and $\phi_{4}$ are a solution to equation (1.12). By (2.29) and Lemma 2.5, $\psi_{3}$ and $\phi_{4}$ are solutions to equation (1.13).

Remark 2.9. Observe that if

$$
\sigma^{\prime \prime} \leq 0 \quad \text { in }(0, \infty)
$$

that is

$$
\operatorname{Ric}_{o}(x) \geq 0 \text { for all } x \in M
$$

or if

$$
\sigma^{\prime \prime} \geq 0 \quad \text { in }(0, \infty)
$$


that is

$$
\operatorname{Ric}_{o}(x) \leq 0 \text { for all } x \in M
$$

then, from (1.5), we can infer that condition $(C)$ with $\rho_{0}=0$ is satisfied.

Remark 2.10. (i) Note that if $\sigma(\rho)=\rho(\rho \geq 0)$ and $\rho_{0}=0$, then, obviously, classical radial solutions to equations (1.12) and (1.13) given above coincide with those determined in [5] for the case $M=\mathbb{R}^{N}$. Note that radial solutions to elliptic equations involving Pucci extremal operators have been also investigated in $[6,11]$.

(ii) Observe that there holds:

$$
\begin{aligned}
& \phi_{1}(\rho)=C_{1} \int_{1}^{\rho} \frac{d \xi}{[S(\xi)]^{\frac{\lambda}{\Lambda}}}+C_{2} \quad\left(\rho>\rho_{0}\right) \\
& \phi_{2}(\rho)=-C_{1} \int_{\rho}^{\infty} \frac{d \xi}{[S(\xi)]^{\frac{\lambda}{\Lambda}}}+C_{2} \quad\left(\rho>\rho_{0}\right) \\
& \phi_{3}(\rho)=-C_{1} \int_{1}^{\rho} \frac{d \xi}{[S(\xi)]^{\frac{\Lambda}{\lambda}}}+C_{2} \quad\left(\rho>\rho_{0}\right) \\
& \phi_{4}(\rho)=C_{1} \int_{\rho}^{\infty} \frac{d \xi}{[S(\xi)]^{\frac{\Lambda}{\lambda}}}+C_{2} \quad\left(\rho>\rho_{0}\right) .
\end{aligned}
$$

In particular, for $\lambda=\Lambda=1$, we have (see (2.26)):

$$
\begin{aligned}
& \phi_{2}(\rho)=-C_{1} G(x, o)+C_{2}, \quad \phi_{4}(\rho)=C_{1} G(x, o)+C_{2} \quad\left(\rho>\rho_{0}\right), \\
& \text { where } \rho \equiv \rho(x)=\operatorname{dist}(x, o) \quad(x \in M \backslash\{o\}) .
\end{aligned}
$$

\section{Hadamard type theorems}

We shall prove a Hadamard three circles type theorem for viscosity solutions to equation (1.1) or (1.2). Such results have been established in [5] for the case $M=\mathbb{R}^{N}$; furthermore, when $M=\mathbb{R}^{N}$ and $\lambda=\Lambda=1$, they can be found in $[14]$.

We have the following

Theorem 3.1. Let hypothesis $(C)$ be satisfied. Let $\rho_{0}<\rho_{2}<\rho_{1}$ and $\Omega \subseteq M$ be a domain containing $\bar{B}_{\rho_{1}}$.

(i) Let $u$ be a viscosity supersolution to equation

$$
\mathcal{P}_{\lambda, \Lambda}^{+}\left(D^{2} u\right)=0 \quad \text { in } \Omega .
$$

Then

$$
m(\rho) \geq \frac{m\left(\rho_{2}\right)\left[-\int_{1}^{\rho} \frac{d \xi}{[\sigma(\xi)]^{\alpha}}+\int_{1}^{\rho_{1}} \frac{d \xi}{[\sigma(\xi)]^{\alpha}}\right]+m\left(\rho_{1}\right)\left[-\int_{1}^{\rho_{2}} \frac{d \xi}{[\sigma(\xi)]^{\alpha}}+\int_{1}^{\rho} \frac{d \xi}{[\sigma(\xi)]^{\alpha}}\right]}{-\int_{1}^{\rho_{2}} \frac{d \xi}{[\sigma(\xi)]^{\alpha}}+\int_{1}^{\rho_{1}} \frac{d \xi}{[\sigma(\xi)]^{\alpha}}}
$$

for all $\rho \in\left[\rho_{2}, \rho_{1}\right]$. 
Moreover, if condition $\left(H_{3}\right)$ is satisfied, then

$$
m(\rho) \geq \frac{m\left(\rho_{2}\right)\left[\int_{\rho}^{\infty} \frac{d \xi}{[\sigma(\xi)]^{\alpha}}-\int_{\rho_{1}}^{\infty} \frac{d \xi}{[\sigma(\xi)]^{\alpha}}\right]+m\left(\rho_{1}\right)\left[\int_{\rho_{2}}^{\infty} \frac{d \xi}{[\sigma(\xi)]^{\alpha}}-\int_{\rho}^{\infty} \frac{d \xi}{[\sigma(\xi)]^{\alpha}}\right]}{\int_{\rho_{2}}^{\infty} \frac{d \xi}{[\sigma(\xi)]^{\alpha}}-\int_{\rho_{1}}^{\infty} \frac{d \xi}{[\sigma(\xi)]^{\alpha}}}
$$

for all $\rho \in\left[\rho_{2}, \rho_{1}\right]$.

(ii) Let $u$ be a viscosity supersolution to equation

$$
\mathcal{P}_{\lambda, \Lambda}^{-}\left(D^{2} u\right)=0 \quad \text { in } \Omega
$$

Then

$$
m(\rho) \geq \frac{m\left(\rho_{2}\right)\left[-\int_{1}^{\rho} \frac{d \xi}{[\sigma(\xi)]^{\beta}}+\int_{1}^{\rho_{1}} \frac{d \xi}{[\sigma(\xi)]^{\beta}}\right]+m\left(\rho_{1}\right)\left[-\int_{1}^{\rho_{2}} \frac{d \xi}{[\sigma(\xi)]^{\beta}}+\int_{1}^{\rho} \frac{d \xi}{[\sigma(\xi)]^{\beta}}\right]}{-\int_{1}^{\rho_{2}} \frac{d \xi}{[\sigma(\xi)]^{\beta}}+\int_{1}^{\rho_{1}} \frac{d \xi}{[\sigma(\xi)]^{\beta}}}
$$

for all $\rho \in\left[\rho_{2}, \rho_{1}\right]$. Moreover, if condition $\left(H_{2}\right)$ is satisfied, then

$$
m(\rho) \geq \frac{m\left(\rho_{2}\right)\left[\int_{\rho}^{\infty} \frac{d \xi}{[\sigma(\xi)]^{\beta}}-\int_{\rho_{1}}^{\infty} \frac{d \xi}{[\sigma(\xi)]^{\beta}}\right]+m\left(\rho_{1}\right)\left[\int_{\rho_{2}}^{\infty} \frac{d \xi}{[\sigma(\xi)]^{\beta}}-\int_{\rho}^{\infty} \frac{d \xi}{[\sigma(\xi)]^{\beta}}\right]}{\int_{\rho_{2}}^{\infty} \frac{d \xi}{[\sigma(\xi)]^{\beta}}-\int_{\rho_{1}}^{\infty} \frac{d \xi}{[\sigma(\xi)]^{\beta}}}
$$

for all $\rho \in\left[\rho_{2}, \rho_{1}\right]$.

Proof. Let

$$
\begin{aligned}
C_{1} & :=\frac{m\left(\rho_{2}\right)-m\left(\rho_{1}\right)}{-\int_{1}^{\rho_{2}} \frac{d \xi}{[\sigma(\xi)]^{\alpha}}+\int_{1}^{\rho_{1}} \frac{d \xi}{[\sigma(\xi)]^{\alpha}}}, \\
C_{2} & :=\frac{m\left(\rho_{2}\right) \int_{1}^{\rho_{1}} \frac{d \xi}{[\sigma(\xi)]^{\alpha}}-m\left(\rho_{1}\right) \int_{1}^{\rho_{2}} \frac{d \xi}{[\sigma(\xi)]^{\alpha}}}{-\int_{1}^{\rho_{2}} \frac{d \xi}{[\sigma(\xi)]^{\alpha}}+\int_{1}^{\rho_{1}} \frac{d \xi}{[\sigma(\xi)]^{\alpha}}} .
\end{aligned}
$$

Clearly, $C_{1}>0$. Moreover, with this choice of $C_{1}$ and $C_{2}$, by Lemma 2.8, $\psi_{1}$ is a subsolution to equation to problem

$$
\begin{cases}\mathcal{P}_{\lambda, \Lambda}^{+}\left(D^{2} u\right)=0 & \text { in } B_{\rho_{1}} \backslash \bar{B}_{\rho_{2}} \\ u=m\left(\rho_{2}\right) & \text { on } \partial B_{\rho_{2}} \\ u=m\left(\rho_{1}\right) & \text { on } \partial B_{\rho_{1}} ;\end{cases}
$$

instead, $u$ is a supersolution to the same problem. By comparison principle,

$$
u \geq \psi_{1} \quad \text { in } B_{\rho_{1}} \backslash \bar{B}_{\rho_{2}} .
$$

Hence (3.40) follows. Analogously it is possible to get the (3.41)-(3.44).

Similarly, it is possible to show next

Theorem 3.2. Let hypothesis $(C)$ be satisfied. Let $\rho_{0}<\rho_{2}<\rho_{1}$ and $\Omega \subseteq M$ be a domain containing $\bar{B}_{\rho_{1}}$. 
(i) Let $u$ be a viscosity subsolution to equation (3.42). Then

$$
M(\rho) \leq \frac{M\left(\rho_{2}\right)\left[\int_{1}^{\rho} \frac{d \xi}{[\sigma(\xi)]^{\alpha}}-\int_{1}^{\rho_{1}} \frac{d \xi}{[\sigma(\xi)]^{\alpha}}\right]+M\left(\rho_{1}\right)\left[\int_{1}^{\rho_{2}} \frac{d \xi}{[\sigma(\xi)]^{\alpha}}-\int_{1}^{\rho} \frac{d \xi}{[\sigma(\xi)]^{\alpha}}\right]}{\int_{1}^{\rho_{2}} \frac{d \xi}{[\sigma(\xi)]^{\alpha}}-\int_{1}^{\rho_{1}} \frac{d \xi}{[\sigma(\xi)]^{\alpha}}}
$$

for all $\rho \in\left[\rho_{2}, \rho_{1}\right]$. Moreover, if condition $\left(H_{3}\right)$ is satisfied, then

$$
M(\rho) \leq \frac{M\left(\rho_{2}\right)\left[-\int_{\rho}^{\infty} \frac{d \xi}{[\sigma(\xi)]^{\alpha}}+\int_{\rho_{1}}^{\infty} \frac{d \xi}{[\sigma(\xi)]^{\alpha}}\right]+M\left(\rho_{1}\right)\left[-\int_{\rho_{2}}^{\infty} \frac{d \xi}{[\sigma(\xi)]^{\alpha}}+\int_{\rho}^{\infty} \frac{d \xi}{[\sigma(\xi)]^{\alpha}}\right]}{-\int_{\rho_{2}}^{\infty} \frac{d \xi}{[\sigma(\xi)]^{\alpha}}+\int_{\rho_{1}}^{\infty} \frac{d \xi}{[\sigma(\xi)]^{\alpha}}}
$$

for all $\rho \in\left[\rho_{2}, \rho_{1}\right]$.

(ii) Let $u$ be a viscosity supersolution to equation (3.39). Then

$$
M(\rho) \leq \frac{M\left(\rho_{2}\right)\left[\int_{1}^{\rho} \frac{d \xi}{[\sigma(\xi)]^{\beta}}-\int_{1}^{\rho_{1}} \frac{d \xi}{[\sigma(\xi)]^{\beta}}\right]+M\left(\rho_{1}\right)\left[\int_{1}^{\rho_{2}} \frac{d \xi}{[\sigma(\xi)]^{\beta}}-\int_{1}^{\rho} \frac{d \xi}{[\sigma(\xi)]^{\beta}}\right]}{\int_{1}^{\rho_{2}} \frac{d \xi}{[\sigma(\xi)]^{\beta}}-\int_{1}^{\rho_{1}} \frac{d \xi}{[\sigma(\xi)]^{\beta}}}
$$

for all $\rho \in\left[\rho_{2}, \rho_{1}\right]$. Moreover, if condition $\left(H_{2}\right)$ is satisfied, then

$$
M(\rho) \leq \frac{M\left(\rho_{2}\right)\left[\int_{\rho}^{\infty} \frac{d \xi}{[\sigma(\xi)]^{\beta}}-\int_{\rho_{1}}^{\infty} \frac{d \xi}{[\sigma(\xi)]^{\beta}}\right]+M\left(\rho_{1}\right)\left[\int_{\rho_{2}}^{\infty} \frac{d \xi}{[\sigma(\xi)]^{\beta}}-\int_{\rho}^{\infty} \frac{d \xi}{[\sigma(\xi)]^{\beta}}\right]}{\int_{\rho_{2}}^{\infty} \frac{d \xi}{[\sigma(\xi)]^{\beta}}-\int_{\rho_{1}}^{\infty} \frac{d \xi}{[\sigma(\xi)]^{\beta}}}
$$

for all $\rho \in\left[\rho_{2}, \rho_{1}\right]$.

Example 3.3. If $\sigma(\rho)=\sinh \rho$, that is $M=\mathbb{H}^{N}$, then Theorems 3.1-3.2 can be applied. Note that in this case

$$
\operatorname{Ric}_{o}(x)=-(N-1)<0 \text { for all } x \in \mathbb{H}^{N} .
$$

Corollary 3.4. Let hypothesis $(C)$ be satisfied.

(i) Let condition $\left(\mathrm{H}_{3}\right)$ be satisfied. Let $u$ be a nonnegative viscosity supersolution to equation (1.1). Then the function

$$
\rho \mapsto \frac{m(\rho)}{\int_{\rho}^{\infty} \frac{d \xi}{[\sigma(\xi)]^{\alpha}}}
$$

is increasing in $\left(\rho_{0}, \infty\right)$.

(ii) Let condition $\left(\mathrm{H}_{2}\right)$ be satisfied. Let $u$ be a nonnegative viscosity subsolution to equation (1.2). Then the function

$$
\rho \mapsto \frac{m(\rho)}{\int_{\rho}^{\infty} \frac{d \xi}{[\sigma(\xi)]^{\beta}}}
$$

is increasing in $\left(\rho_{0}, \infty\right)$. 
Proof. (i) Let $\rho_{0}<\rho_{2}<\rho_{1}, \rho \in\left[\rho_{2}, \rho_{1}\right]$. Since $m\left(\rho_{1}\right) \geq 0$, sending $\rho_{1} \rightarrow \infty$ in inequality (3.41) we get

$$
m(\rho) \geq \frac{m\left(\rho_{2}\right) \int_{\rho}^{\infty} \frac{d \xi}{[\sigma(\xi)]^{\beta}}}{\int_{\rho_{2}}^{\infty} \frac{d \xi}{[\sigma(\xi)]^{\beta}}} \quad\left(\rho \geq \rho_{2}\right),
$$

whence the thesis. The proof of $(i i)$ is analogous.

\section{Liouville type theorem}

We shall prove the following

Theorem 4.1. Let hypotheses $(C)$ and $\left(H_{1}\right)$ be satisfied.

(i) If $u$ is a viscosity supersolution bounded from below to equation

$$
\mathcal{P}_{\lambda, \Lambda}^{+}\left(D^{2} u\right)=0 \quad \text { in } M
$$

then $u$ is constant.

(ii) If $u$ is a viscosity subsolution bounded from above to equation

$$
\mathcal{P}_{\lambda, \Lambda}^{-}\left(D^{2} u\right)=0 \quad \text { in } M
$$

then $u$ is constant.

Proof. (i) By Theorem 3.1-(i), inequality (3.40) is satisfied for any $\rho_{1} \geq \rho \geq$ $\rho_{2}>\rho_{0}$. Letting $\rho_{1} \rightarrow \infty$ in (3.40), obtains

$$
m(\rho) \geq m\left(\rho_{2}\right) \text { for all } \rho \geq \rho_{2}>\rho_{0},
$$

since hypothesis $\left(H_{1}\right)$ holds true and $m \in L^{\infty}((0, \infty))$, for $u$ is bounded from below.

Since $\rho \mapsto m(\rho)$ is decreasing in $(0, \infty)$, we can infer that

$$
m(\rho)=m\left(\rho_{0}\right)=u\left(x_{0}\right) \text { for any } \rho>\rho_{0}
$$

for some $x_{0} \in \bar{B}_{\rho_{0}}$. Hence, for any $\rho>\rho_{0}$ we have:

$$
\min _{\bar{B}_{\rho}} u=u\left(x_{0}\right)
$$

furthermore, $x_{0}$ is an interior point of $\bar{B}_{\rho}$, since $x_{0} \in \bar{B}_{\rho_{0}} \subset B_{\rho}$.

For any $\rho>\rho_{0}$, by Proposition 2.3 with $\Omega=B_{\rho}$ it follows that

$$
u \equiv u\left(x_{0}\right) \text { in } B_{\rho} .
$$

Since $\rho>0$ is arbitrary, the thesis follows. Similarly, we can get the thesis in case $(i i)$.

Remark 4.2. (i) In Theorem 4.1, instead of $u$ bounded from below, we can require

$$
\liminf _{\rho \rightarrow \infty} \frac{m(\rho)}{\int_{1}^{\rho} \frac{d \xi}{[\sigma(\xi)]^{\alpha}}} \geq 0
$$


moreover, instead of $u$ bounded from above, we can require

$$
\limsup _{\rho \rightarrow \infty} \frac{M(\rho)}{\int_{1}^{\rho} \frac{d \xi}{[\sigma(\xi)]^{\alpha}}} \leq 0 .
$$

These growth conditions are optimal (indeed, see Remark 1 [5], for the special case $\sigma(\rho)=\rho(\rho \geq 0))$.

(ii) A Liouville type theorem does not hold neither for viscosity supersolutions to equation (1.1), nor for viscosity subsolutions to equation (1.2) (indeed, see Remark 3 in [5], for the special case $\sigma(\rho)=\rho(\rho \geq 0)$ ).

Remark 4.3. (i) If $\lambda=\Lambda=1$, results contained in Theorem 4.1 and Remark 2.6 are in agreement with those given in [8].

(ii) If $M=\mathbb{R}^{N}$, since $\sigma(\rho)=\rho(\rho \geq 0)$, results contained in Theorem 4.1 are in agreement with those established in [5].

Example 4.4. (i) If $M=\mathbb{H}^{N}$, then Theorem 4.1 cannot be applied, since $\left(H_{1}\right)$ is not satisfied. Indeed, for $\lambda=\Lambda=1$, its conclusion is false (see $[8])$.

(ii) If $S(\rho) \leq C \rho^{k}$ for all $\rho \geq \rho$ for some $C>0$ and $\rho>0$, with $k \leq \frac{\Lambda}{\lambda}$, then Theorem 4.1 can be applied.

\section{Liouville type theorem for elliptic equations with zero order terms}

Now we prove a Liouville type theorem for equation (1.3), supposing that $F \in C(M \times \mathcal{E} ; \mathbb{R})$ satisfies $(2.27)-(2.28)$.

Consider $\mathbb{R}^{N}$ as a model manifold with metric given by (1.4) with $\sigma$ replaced by $\psi(\rho):=\rho(\rho \geq 0)$. Clearly, the Ricci curvature of $\mathbb{R}^{N}$ is $-(N-1)$ $\frac{\psi^{\prime \prime}(\rho)}{\psi(\rho)}=0$ for all $\rho>0$. Hence, if assumption $\left(H_{4}\right)$ is satisfied, from the socalled Laplacian comparison theorem (see, e.g., Proposition 2.15 in [7]) we get

$$
\frac{\sigma^{\prime}(\rho)}{\sigma(\rho)} \leq \frac{\psi^{\prime}(\rho)}{\psi(\rho)}=\frac{1}{\rho} \quad \text { for all } \rho>0
$$

Moreover, note that $\left(H_{4}\right)$ implies that there exist $C_{1}>0$ and $\bar{\rho}>0$ such that

$$
\sigma(\rho) \leq C_{1} \rho \text { for all } \rho>\bar{\rho}
$$

So

$$
\int_{\rho}^{\infty} \frac{d \xi}{\xi^{\beta}} \leq C_{1}^{\beta} \int_{\rho}^{\infty} \frac{d \xi}{[\sigma(\xi)]^{\beta}}<\infty \quad(\rho>\bar{\rho}) .
$$

This implies

$$
\beta>1
$$

We shall prove the following 
Theorem 5.1. Let hypotheses $\left(H_{2}\right)$ and $\left(H_{4}\right)$ be satisfied. Let $h \in C(M ;[0, \infty))$; let there exist $H \geq 0, \gamma \geq-2$ and $\rho>0$ such that

$$
h(x) \geq H \rho^{\gamma} \text { for all } x \equiv(\rho, \theta) \text { with } \rho \geq \underline{\rho} .
$$

Let $u \geq 0$ be a viscosity supersolution to equation

$$
F\left(x, D^{2} u\right)+h(x) u^{p}=0 \quad \text { in } M .
$$

If

$$
\gamma=-2 \text { and } 0<p<1
$$

or

$$
\gamma>-2 \text { and } 0<p<\frac{\beta+1+\gamma}{\beta-1}
$$

then

$$
u \equiv 0 \quad \text { in } M \text {. }
$$

Proof. Since $u \geq 0$ in $M$, from Proposition 2.3 we can infer that if there exists $x_{0} \in M$ such that $u\left(x_{0}\right)=0$, then $u \equiv 0$ in $M$. Hence we suppose, by contradiction, that $u>0$ in $M$. Thus $m>0$ in $[0, \infty)$; furthermore, again by Proposition 2.3, $m$ is strictly decreasing in $(0, \infty)$.

Since $u$ is a positive supersolution to equation (1.2), by Corollary 3.4,

$$
m(r) \leq \frac{m(R) \int_{r}^{\infty} \frac{d \xi}{[\sigma(\xi)]^{\beta}}}{\int_{R}^{\infty} \frac{d \xi}{[\sigma(\xi)]^{\beta}}} \text { for all } R \geq r>0
$$

Take $R>r \geq r_{0}>0$; then define

$$
\zeta(\rho):=m(r)\left\{1-\frac{\left[(\rho-r)^{+}\right]^{3}}{(R-r)^{3}}\right\} \quad(\rho>0) .
$$

Note that

$$
\zeta(x) \leq 0<u(x) \text { for any } x \equiv(\rho, \theta) \in M \text { with } \rho \geq R,
$$

and

$$
\zeta(x) \equiv m(r)<u(x) \quad \text { for any } \quad x \equiv(\rho, \theta) \in M \text { with } \rho<r .
$$

Furthermore, there exists $x_{0} \in \partial B_{r}$ such that

$$
\zeta\left(x_{0}\right)=u\left(x_{0}\right) .
$$

Hence

$$
0 \geq \min _{M}(u-\zeta)=u(\bar{x})-\zeta(\bar{x}) \quad \text { with } \bar{x} \equiv(\bar{\rho}, \bar{\theta}), r \leq \bar{\rho}<R .
$$

Using $\zeta$ as test function in the definition of viscosity solution to equation (1.3) (see Definition 2.1), by (2.34) we get

$$
\mathcal{P}_{\lambda, \Lambda}^{-}\left(D^{2} \zeta(\bar{x})\right)+h(\bar{x})[u(\bar{x})]^{p} \leq 0 .
$$


We have, in $M \backslash\{o\}$,

$$
\mathcal{P}_{\lambda, \Lambda}^{-}\left(D^{2} \zeta(x)\right)=-\frac{3 \Lambda m(r)}{(R-r)^{3}}\left[2+(N-1)(\rho-r)^{+} \frac{\sigma^{\prime}(\rho)}{\sigma(\rho)}\right](\rho-r)^{+} .
$$

By (5.59), (5.54) and (5.51),

$$
\begin{aligned}
{[u(\bar{x})]^{p} } & \leq \frac{3 \Lambda m(r)}{H(R-r)^{3}}\left[2+(N-1)(\bar{\rho}-r)^{+} \frac{\sigma^{\prime}(\bar{\rho})}{\sigma(\bar{\rho})}\right](\bar{\rho}-r)^{+} \bar{\rho}^{-\gamma} \\
& \leq \frac{3 \Lambda m(r)}{H(R-r)^{3}}\left[2+(N-1) \frac{(\bar{\rho}-r)^{+}}{\bar{\rho}}\right](\bar{\rho}-r)^{+} \bar{\rho}^{-\gamma}
\end{aligned}
$$

If $\bar{\rho}=r$, then $u(\bar{x})=0$; this is a contradiction. Therefore, we can suppose that $r<\bar{\rho}<R$. So, from (5.61) it follows

$$
[u(\bar{x})]^{p} \leq \frac{3 \Lambda(N+1) \bar{\rho}^{-\gamma} m(r)}{H(R-r)^{2}} .
$$

Since $u(\bar{x}) \geq m(R)$ and $r<\bar{\rho}<R$, we have

$$
m(R) \leq C\left(\frac{R^{\gamma^{-}} m(r)}{r^{\gamma^{+}}(R-r)^{2}}\right)^{\frac{1}{p}}
$$

for all $R>r \geq r_{0}$ with $C=\left(\frac{3 \Lambda(N+1)}{H}\right)^{\frac{1}{p}}$.

From (5.63) and (5.58) we deduce that

$$
m(R) \leq C\left(\frac{R^{\gamma^{-}} \int_{r}^{\infty} \frac{d \xi}{[\sigma(\xi)]^{\beta}} m(R)}{r^{\gamma^{+}} \int_{R}^{\infty} \frac{d \xi}{[\sigma(\xi)]^{\beta}}(R-r)^{2}}\right)^{\frac{1}{p}}
$$

for all $R>r \geq r_{0}$. Inequality (5.64) with $r=\frac{R}{2}$ yields:

$$
m(R) \leq C\left(\frac{R^{\gamma^{-}} \int_{R / 2}^{\infty} \frac{d \xi}{[\sigma(\xi)]^{\beta}} m(R)}{\left(\frac{R}{2}\right)^{\gamma^{+}} \int_{R}^{\infty} \frac{d \xi}{[\sigma(\xi)]^{\beta}}\left(\frac{R}{2}\right)^{2}}\right)^{\frac{1}{p}} .
$$

By $\left(H_{4}\right)$ and $(1.5)$,

$$
\sigma\left(\frac{\rho}{2}\right) \geq \frac{1}{2} \sigma(0)+\frac{1}{2} \sigma(\rho)=\frac{1}{2} \sigma(\rho) \quad(\rho>0) .
$$

Observe that

$$
\frac{\int_{R / 2}^{\infty} \frac{d \xi}{[\sigma(\xi)]^{\beta}}}{\int_{R}^{\infty} \frac{d \xi}{[\sigma(\xi)]^{\beta}}}=2 \frac{\int_{R}^{\infty} \frac{d \xi}{\left[\sigma\left(\frac{\xi}{2}\right)\right]^{\beta}}}{\int_{R}^{\infty} \frac{d \xi}{[\sigma(\xi)]^{\beta}}} \leq 2^{\beta+1} \frac{\int_{R}^{\infty} \frac{d \xi}{[\sigma(\xi)]^{\beta}}}{\int_{R}^{\infty} \frac{d \xi}{[\sigma(\xi)]^{\beta}}}=2^{\beta+1} ;
$$

here use of (5.66) has been made. This combined with (5.65) yields

$$
m(R) \leq C \frac{[m(R)]^{\frac{1}{p}}}{R^{\frac{\gamma+2}{p}}} \text { for all } R>2 r_{0},
$$


where the same $C$ denotes from now on different positive constants independent of $R$.

If $\gamma=-2$ and $0<p<1$, then the thesis follows by the same arguments as in the proof of Theorem 4.1 in [5].

Suppose now $\gamma>-2$ and $0<p<\frac{\beta+1+\gamma}{\beta-1}$. If $0<p \leq 1$, from (5.67) we can infer that

$$
R^{\frac{\gamma+2}{p}} \leq C[m(R)]^{\frac{1-p}{p}} \leq C[m(0)]^{\frac{1-p}{p}} \text { for all } R>2 r_{0} .
$$

Sending $R \rightarrow \infty$, we have a contradiction. Thus suppose $p>1$. From (5.67) we deduce

$$
m(R) \leq \frac{C}{R^{\frac{\gamma+2}{p-1}}} \quad \text { for all } R>2 r_{0}
$$

Hence

$$
\frac{m(R)}{\int_{R}^{\infty} \frac{d \xi}{[\sigma(\xi)]^{\beta}}} \leq \frac{C}{R^{\frac{\gamma+2}{p-1}} \int_{R}^{\infty} \frac{d \xi}{[\sigma(\xi)]^{\beta}}} .
$$

We claim that

$$
\lim _{R \rightarrow \infty} R^{\frac{\gamma+2}{p-1}} \int_{R}^{\infty} \frac{d \xi}{[\sigma(\xi)]^{\beta}}=\infty .
$$

In fact, by (5.52) and (5.53)

$$
R^{\frac{\gamma+2}{p-1}} \int_{R}^{\infty} \frac{d \xi}{[\sigma(\xi)]^{\beta}} \geq \frac{1}{C_{1}^{\beta}(\beta-1)} R^{\frac{\gamma+2}{p-1}-\beta+1} \rightarrow \infty \quad \text { as } R \rightarrow \infty .
$$

By $(5.69)$

$$
\lim _{R \rightarrow \infty} \frac{m(R)}{\int_{R}^{\infty} \frac{d \xi}{[\sigma(\xi)]^{\beta}}}=0
$$

this is in contrast with the fact the function $R \mapsto \frac{m(R)}{\int_{R}^{\infty} \frac{d \xi}{[\sigma(\xi)]^{\beta}}}$ is positive and increasing in $(0, \infty)$ (see Corollary 3.4). Thus the proof is complete.

Remark 5.2. Concerning hypothesis $\left(H_{4}\right)$ in Theorem 5.1, observe that on $\mathbb{H}^{N}$, for which $\sigma^{\prime \prime}>0$ (see Subsection 2.1), the conclusion fails for $\lambda=\Lambda=1$ under proper restrictions on $p$. Indeed, in [12] it is shown that equation

$$
\Delta u+u^{p}=0 \text { in } \mathbb{H}^{N}
$$

admits a positive solution, if $p>1$ and $N=2$, or if $1<p<\frac{N+2}{N-2}$ and $N \geq 3$.

Remark 5.3. (i) When $M=\mathbb{R}^{N}$ Theorem 5.1 is in agreement with Theorem 4.1 in [5]. In [5] also the case

$$
\gamma>-2, \quad p=\frac{\beta+1+\gamma}{\beta-1}
$$

is included; it is an open problem to understand if the same can be made in the present situation, too. 
(ii) As observed in Remark 7 in [5], equation (1.3) when $M=\mathbb{R}^{N}$ admits a nontrivial classical solution, if $\gamma<-2$ and $p>0$, or $\gamma=-2$ and $p \geq 1$, or $\gamma>-2$ and $p>\frac{\beta+1-\gamma}{\beta-1}$.

\section{References}

[1] Azagra, D., Ferrera, J., Sanz, B.: Viscosity solutions to second order partial differential equations on Riemannian manifolds. J. Diff. Equ. 245, 307-336 (2008)

[2] Birindelli, I., Capuzzo-Dolcetta, I., Cutrì, A.: Liouville theorems for semilinear equations on the Heisenberg group. Ann. Inst. Henri Poincar Anal. Nonlinear 14, 295-308 (1997)

[3] Cabré, X.: Nondivergent elliptic equations on manifolds with nonnegative curvature. Comm. Pure Appl. Math. 50, 623-665 (1997)

[4] Capuzzo-Dolcetta, I., Cutrì, A.: Hadamard and Liouville type results for fully nonlinear partial differential inequalities. Comm. Cont. Math. 3, 435-448 (2003)

[5] Cutrì, A., Leoni, F.: On the Liouville property for fully nonlinear equations. Ann. Inst. Henri Poincar Anal. Nonlinear 17, 219-245 (2000)

[6] Felmer, P., Quaas, A.: Positive radial solutions to a semilinear equation involving the Pucci's operator. J. Diff. Equ. 199, 376-393 (2004)

[7] Greene, R., Wu, W.: Function Theory of Manifolds Which Posses a Pole. Springer, Berlin (1979)

[8] Grigoryan, A.: Analytic and geometric background of recurrence and nonexplosion of the Brownian motion on Riemannian manifolds. Bull. Am. Math. Soc. 36, 135-249 (1999)

[9] Grigoryan, A.: Heat Kernel and Analysis on Manifold. American Mathematical Society-International Press, Somerville (2009)

[10] Kim, S.: Harnack inequality for nondivergent elliptic operators on Riemannian manifolds. Pacific J. Math. 213, 281-293 (2004)

[11] Li, W., Cheng, J.: Nonnegative radial solutions to a class of elliptic equations involving the Pucci's extremal operator. J. Math. Anal. Appl. 341, 386397 (2008)

[12] Mancini, G., Sandeep, K.: On a semilinear elliptic equation on $\mathbb{H}^{n}$. Ann. Sci. Normale Suppl. 7, 635-671 (2008)

[13] Peng, S., Zhou, D.: Maximum principles for viscosity solutions on Riemannian manifolds. (2011) (preprint)

[14] Protter, M.H., Weinberger, H.F.: Maximum Principles in Differential Equations. Prentice-Hall, Englewood Cliffs (1967)

[15] Rigoli, M., Setti, A.G.: Liouville type theorems for $\varphi$-subharmonic functions. Rev. Mat. Iberoam. 17, 471-520 (2001) 
[16] Stroock, D.W.: Non-divergence form operators and variations on Yau's explosion criterion. J. Funct. Anal. Appl. 4, 565-574 (1998)

[17] Wang, Y., Zhang, X.: Measure estimates, Harnack inequalities and Ricci lower bound (2011) (preprint)

\section{Fabio Punzo}

Dipartimento di Matematica "G. Castelnuovo"

Università di Roma "La Sapienza"

P.le A. Moro 5

00185 Rome

Italy

e-mail:punzo@mat.uniroma1.it

Received: 13 April 2012.

Accepted: 4 November 2012. 